\title{
Discrete convolution operators in positive characteristic: a variation on the Floquet-Bloch Theory
}

\author{
Mikhail Zaidenberg
}

\begin{abstract}
The classical Floquet theory deals with Floquet-Bloch solutions of periodic PDEs, see e.g. $\mathbf{K} \mathbf{u}_{3}$. A discrete version of this theory for difference vector equations on lattices, including the Floquet theory on infinite periodic graphs, was developed by Peter Kuchment $\mathbf{K} \mathbf{u}_{1}, \mathbf{K u}_{2}$. Here we propose a variation on this theory for matrix convolution operators acting on vector functions on lattices with values in a field of positive characteristic.
\end{abstract}

\section{Introduction}

The classical Floquet theory founded in the work of Hill, Floquet, Lyapunov, and Felix Bloch, deals with different types of periodic ordinary differential equations. An advanced version of the theory applies to the periodic PDEs and deals with the Floquet-Bloch solutions $\mathbf{K u}_{3}$. A discrete analog appeals to the notion of a periodic graph, see e.g. [Co, $\mathbf{v B}$. This is an infinite graph equipped with a free action of a free abelian group (a lattice) $\Lambda$ of finite rank. Peter Kuchment $\mathbf{K u}_{1}-\mathbf{K u}_{2}$ adopted the Floquet theory for a periodic difference operator $\Delta$ (e.g., the Laplace, Schrödinger, Markov operator, etc.) acting in a translation invariant function space on a periodic graph $\Gamma$; see also $\mathbf{K u P i}$, $\mathbf{K u V a}$. Assume that a lattice $\Lambda$ acts on $\Gamma$ with a finite number of orbits. Then the following hold.

(1) Consider a periodic difference equation $\Delta(f)=0$ on an infinite periodic graph $\Gamma$. There exists a sequence $\left(f_{n}\right)$ of finite linear combinations of Floquet solutions such that every other solution $f$ can be decomposed in a series $f=\sum_{n=1}^{\infty} a_{n} f_{n}$ with constant coefficients.

(2) Every $l_{2}$-solution can be approximated by solutions with finite support.

(3) A criterion is provided as to when this equation has no solution.

In these notes we propose a version of the discrete Floquet-Bloch theory in positive characteristic. Our approach is purely algebraic and ignores the analytic aspects, while preserving the main features of the classical theory. In some respect, the Floquet-Bloch theory for discrete operators gains a simplification when passing to a positive characteristic. In particular, the spectral theory occurs to be quite elementary. We consider matrix convolution operators acting on vector functions on a lattice $\Lambda$. Again, this includes the case of a periodic graph. As a motivation we address the survey $\mathbf{Z a}_{3}$.

2010 Mathematics Subject Classification: 11C99, 11T99, 31C20, 37B15, 43A60.

Key words: convolution operator, lattice, finite field, discrete Fourier transform, discrete harmonic function, pluriperiodic function.

Acknowledgements: This is my pleasure to thank Peter Kuchment for informing in his work and useful discussions. 
In positive characteristic, the traditional translation invariant function spaces of the FloquetBloch theory (e.g., the span of all Floquet functions) coincide with the space of all pluri-periodic vector functions (see 2.4 and 2.5). This issue, of course, simplifies things. However, in positive characteristic the latter space is not well adopted for applying the Fourier transform 1 . More suitable in this respect is the subspace $\mathcal{F}_{p}(\Lambda, \mathbb{K})$, though it is characteristic-dependent. It consists of all pluriperiodic vector functions on $\Lambda$ with values in the base field $\mathbb{K}$ whose period sublattices $\Lambda^{\prime} \subseteq \Lambda$ are of finite indices coprime to $p$. The role of Bloch functions is played now by elementary functions. Such a function is a product of a constant vector and a character of $\Lambda$ with values in the multiplicative group $\mathbb{K}^{\times}$of $\mathbb{K}$.

Besides this Introduction, the paper contains sections 2-5. After recalling in section 2 necessary preliminaries on harmonic analysis on lattices in positive characteristic, we introduce our principal function space and define the Fourier transform. The rest of the paper is devoted to spectral analysis of convolution operators. In section 3 we treat the scalar case, where such operators are semi-simple (i.e. diagonalizable, see Propositions 3.2 and 3.5). While in the vector case (see section 4) these operators are not any more semi-simple, in general. Given a convolution operator $\Delta$ acting on vector functions, we construct in Theorems 4.2 and 4.7 a Jordan basis of $\Delta$ consisting of elementary functions. Until subsection 4.3 the base field $\mathbb{K}$ is assumed being algebraically closed. In subsection 4.3 we deduce, for a generalized Laplace equation $\Delta(f)=0$, the existence of a solution with values in a finite field assuming that there exists such a solution with values in its algebraic closure. In concluding section 5 we provide some examples.

The author is grateful to the referee for correcting an error in a formula in the first draft of the paper.

\section{Preliminaries}

2.1. Harmonic analysis on lattices. Let us recall some generalities; see e.g., $\mathbf{N i}, \mathbf{Z \mathbf { a } _ { 2 }}$ for details.

Given a prime integer $p>0$ we consider the finite Galois field $\mathbb{F}_{p}=\operatorname{GF}(p)$ and its algebraic closure $\mathbb{K}=\mathbb{K}_{p}$. Recall that the multiplicative group $\mathbb{K}^{\times}$of $\mathbb{K}$ is a torsion group. Moreover, every finite subgroup of $\mathbb{K}^{\times}$is cyclic of order coprime to $p$, and every cyclic group $\mathbb{Z} / n \mathbb{Z}$ of order coprime to $p$ is isomorphic to a unique subgroup of $\mathbb{K}^{\times}$; see e.g., $[\mathbf{L N}$.

2.1. Lattices and dual tori. Consider a lattice $\Lambda$ of rank $s>0$. By a character of $\Lambda$ over $\mathbb{K}$ we mean a rational representation $\chi: \Lambda \rightarrow \mathrm{GL}(1, \mathbb{K}) \cong \mathbb{K}^{\times}$. The image $\chi(\Lambda) \subseteq \mathbb{K}^{\times}$is a cyclic subgroup of order coprime to $p$. The dual group $\Lambda^{\vee}$ of $\Lambda$ over $\mathbb{K}$ is the group of all characters $\Lambda \rightarrow \mathbb{K}^{\times}$. It is isomorphic to the algebraic torus $\mathbb{T}^{s}=\left(\mathbb{K}^{\times}\right)^{s}$. By the duality theorem $\left(\Lambda^{\vee}\right)^{\vee} \cong \Lambda$. Indeed, the natural duality pairing $\Lambda \times \Lambda^{\vee} \rightarrow \mathbb{K}^{\times}$is non-degenerate.

More specifically, given a base $\mathcal{V}=\left(v_{1}, \ldots, v_{s}\right)$ of $\Lambda$ we can identify $\Lambda$ with the standard integer lattice $\mathbb{Z}^{s}$ by sending $v=\sum_{i=1}^{s} \lambda_{i} v_{i} \in \Lambda$ to $\lambda(v)=\left(\lambda_{1}, \ldots, \lambda_{s}\right) \in \mathbb{Z}^{s}$. The pairing becomes now

$$
\mathbb{Z}^{s} \times \mathbb{T}^{s} \rightarrow \mathbb{K}^{\times}, \quad(\lambda, z) \longmapsto z^{\lambda}=\prod_{i=1}^{s} z_{i}^{\lambda_{i}} \quad \text { for } \quad z=\left(z_{1}, \ldots, z_{n}\right) \in \mathbb{T}^{s}
$$

Fixing $z \in \mathbb{T}^{s}$ gives a character $\mathbb{Z}^{s} \rightarrow \mathbb{K}^{\times}, \lambda \longmapsto z^{\lambda}$, and every character $\mathbb{Z}^{s} \rightarrow \mathbb{K}^{\times}$arises in this way. On the other hand, every character of the torus $\mathbb{T}^{s}$ with values in $\mathbb{K}^{\times}$is given by a Laurent monomial $z \longmapsto z^{\lambda}$. This yields an identification $\left(\Lambda^{\vee}\right)^{\vee} \cong \Lambda$.

\footnotetext{
${ }^{1}$ Instead of the Floquet transform as exploited in $\mathbf{K} \mathbf{u}_{1}, \mathbf{K u}_{2}$, we apply the usual Fourier transform.
} 
2.2. Convolution operators. Consider the group algebra $\mathbb{K}[\Lambda]$ over $\mathbb{K}$ i.e., the algebra of functions $\Lambda \rightarrow \mathbb{K}$ with finite support endowed with the convolution

$$
(f, g) \longmapsto f * g, \quad \text { where } \quad(f * g)(u)=\sum_{v \in \Lambda} f(v) g(u-v) .
$$

A convolution operator

$$
\Delta_{a} \in \operatorname{End}(\mathbb{K}[\Lambda]), \quad \Delta_{a}(f)=a * f, \quad \text { where } \quad a \in \mathbb{K}[\Lambda],
$$

can be written as

$$
\Delta_{a}=\sum_{v \in \Lambda} a(v) \tau_{-v}
$$

where $\tau_{v}$ denotes the shift by $v: \tau_{v}(f)(u)=f(u+v)$. Clearly, $\Delta_{a}$ commutes with shifts: $\left[\Delta_{a}, \tau_{v}\right]=0$ $\forall v \in \Lambda$. In fact, any endomorphism $\Delta \in \operatorname{End}_{\mathbb{K}}(\mathbb{K}[\Lambda])$ commuting with shifts is a convolution operator with kernel $a=\Delta\left(\delta_{0}\right)$, where $\delta_{v} \in \mathbb{K}[\Lambda]$ stands for the delta-function concentrated on $v \in \Lambda$ i.e.,

$$
\delta_{v}(v)=1 \quad \text { and } \quad \delta_{v}(u)=0 \forall u \neq v .
$$

For $a=\delta_{v}$ we obtain $\Delta_{a}=\tau_{-v}$; indeed, $\delta_{v} * \delta_{0}=\delta_{v}=\tau_{-v}\left(\delta_{0}\right)$. The $\mathbb{K}$-algebra of convolution operators is naturally isomorphic to the group algebra $\mathbb{K}[\Lambda]$.

\subsection{Translation invariant function spaces.}

2.3. Some characteristic-free function spaces. Consider the space $\mathcal{F}=\mathcal{F}(\Lambda, \mathbb{K})$ of all $\mathbb{K}$-valued functions on $\Lambda$. This is a $\mathbb{K}[\Lambda]$-module with respect to the natural action $\mathbb{K}[\Lambda] \times \mathcal{F} \rightarrow \mathcal{F},(a, f) \longmapsto$ $a * f=\Delta_{a}(f)$. A subspace $E \subseteq \mathcal{F}$ is a $\mathbb{K}[\Lambda]$-submodule if and only if it is translation invariant i.e., $\tau_{v}(E)=E$ for all $v \in \Lambda$. A one-dimensional subspace of $\mathcal{F}$ is a $\mathbb{K}[\Lambda]$-submodule if and only if it is spanned by a character $\chi \in \Lambda^{\vee}$. The following function spaces are translation invariant. They arise naturally in the framework of the Floquet-Bloch Theory, cf. $\mathbf{K u}_{1}, \mathbf{K u}_{2}$.

Examples 2.4. $\quad$ (i) The subspace $\mathbb{K}[\Lambda] \subseteq \mathcal{F}$ of all functions with finite support.

(ii) Given a sublattice $\Lambda^{\prime} \subseteq \Lambda$, we let $\mathcal{F}_{\Lambda^{\prime}}$ denote the subspace of all $\Lambda^{\prime}$-periodic functions i.e.,

$$
\mathcal{F}_{\Lambda^{\prime}}=\left\{f \in \mathcal{F} \mid \Lambda(f) \supseteq \Lambda^{\prime}\right\},
$$

where

$$
\Lambda(f)=\left\{v \in \Lambda \mid \tau_{v}(f)=f\right\}
$$

stands for the period lattice of $f$.

(iii) Given an isomorphism $\Lambda \cong \mathbb{Z}^{s}$ we define the subspace $E_{1} \subseteq \mathcal{F}$ of all traces of polynomial functions i.e.,

$$
E_{1}=\left\{q\left|\mathbb{Z}^{s} \in \mathcal{F}\right| q \in \mathbb{K}\left[x_{1}, \ldots, x_{s}\right]\right\} .
$$

(iv) The subspace $E_{2} \subseteq \mathcal{F}$ of all pluri-periodic functions,

$$
E_{2}=\bigcup_{\Lambda^{\prime} \subseteq \Lambda, \operatorname{ind}_{\Lambda} \Lambda^{\prime}<\infty} \mathcal{F}_{\Lambda^{\prime}},
$$

is clearly translation invariant.

(v) The subspace $E_{3} \subseteq \mathcal{F}$ of all polynomially periodic functions. Given an isomorphism $\Lambda \cong \mathbb{Z}^{s}$, a function $g \in \mathcal{F}$ is called polynomially periodic if there exists a sublattice $\Lambda^{\prime} \subseteq \Lambda$ of finite index and a set of polynomials $q_{\bar{v}} \in \mathbb{K}\left[x_{1}, \ldots, x_{s}\right]$, where $\bar{v} \in \Lambda / \Lambda^{\prime}$, such that

$$
g(v)=q_{\bar{v}}(v) \quad \text { if } \quad v \in \bar{v} .
$$


(vi) The subspace $E_{4} \subseteq \mathcal{F}$ of all Floquet functions:

$$
E_{4}=\left\{f=\sum_{i=1}^{n} \chi_{i} g_{i} \in \mathcal{F} \mid \chi_{i} \in \Lambda^{\vee}=\operatorname{Char}\left(\Lambda, \mathbb{K}^{\times}\right), g_{i} \in E_{3}\right\} .
$$

(vii) The subspace $E_{5} \subseteq \mathcal{F}$ spanned by all $\Lambda^{\prime}$-automorphic functions on $\Lambda$, where $\Lambda^{\prime}$ runs over the set of all finite index sublattices of $\Lambda$. Given a sublattice $\Lambda^{\prime} \subseteq \Lambda$, a function $f \in \mathcal{F}$ is called $\Lambda^{\prime}$-automorphic if, for some character $\chi$ of $\Lambda^{\prime}$,

$$
\left(\tau_{u} f\right)(v)=\chi(u) f(v) \quad \forall u \in \Lambda^{\prime}, \forall v \in \Lambda .
$$

(viii) The subspace $E_{6} \subseteq \mathcal{F}$ of all finite-valued functions on $\Lambda$, etc.

Due to the following proposition, there are just three different spaces among the function spaces $E_{1}, \ldots, E_{6}$. In particular, in positive characteristic the Floquet functions are the same as the pluriperiodic functions.

Proposition 2.5. We have

$$
E_{1} \varsubsetneqq E_{2}=E_{3}=E_{4}=E_{5} \varsubsetneqq E_{6} .
$$

Proof. Letting $p=\operatorname{Char}(\mathbb{K})$ we observe that $(x+p \lambda)^{n}=x^{n}$ for every monomial $x^{n}=\prod_{i=1}^{s} x_{i}^{n_{i}}$ (regarded as a function in $x \in \mathbb{Z}^{s}=\Lambda$ ) and for every $\lambda \in \mathbb{Z}^{s}$. Hence every polynomial function on $\Lambda$ is $\Lambda^{\prime}$-periodic, where $\Lambda^{\prime}=p \Lambda$. Therefore

$$
E_{1} \subseteq \mathcal{F}_{\Lambda^{\prime}} \subseteq E_{2} .
$$

By a similar reason, $E_{3}, E_{4} \subseteq E_{2}$. For instance, if $g \in E_{3}$ is as in (v) then $p \Lambda^{\prime} \subseteq \Lambda(g)$. Indeed, for every $v \in \Lambda$ and $v^{\prime} \in \Lambda^{\prime}$ we have $v+p v^{\prime}+\Lambda^{\prime}=v+\Lambda^{\prime}$. Hence

$$
g\left(v+p v^{\prime}\right)=q_{\overline{v+p v^{\prime}}}\left(v+p v^{\prime}\right)=q \overline{v+p v^{\prime}}(v)=q_{\bar{v}}(v)=g(v) .
$$

Thus $E_{3} \subseteq E_{2}$.

It is easily seen that the sum and the product of pluri-periodic functions are again pluri-periodic. Since every character $\chi \in \Lambda^{\vee}$ is $\Lambda_{0}$-periodic, where the sublattice $\Lambda_{0}=\operatorname{ker}(\chi)$ is of finite index in $\Lambda$, the inclusion $E_{4} \subseteq E_{2}$ holds. The inclusions $E_{2} \subseteq E_{3} \subseteq E_{4}$ are evident. It follows that $E_{1} \subseteq E_{2}=E_{3}=E_{4} \subseteq E_{6}$.

Every pluri-periodic function $f \in E_{2}$ (in particular, every character of $\Lambda$ ) is $\Lambda(f)$-automorphic. Hence $E_{2} \subseteq E_{5}$. Let furthermore $f \in E_{5}$ be a $\Lambda^{\prime}$-automorphic function as in (viii), where $\Lambda^{\prime} \subseteq \Lambda$ is a finite index sublattice. Since the period lattice $\Lambda(f)$ contains the finite index sublattice $\Lambda_{0}=$ $\operatorname{ker}(\chi) \subseteq \Lambda^{\prime} \subseteq \Lambda, f$ is pluri-periodic. Thus $E_{2}=E_{5}$ and so $E_{1} \subseteq E_{2}=E_{3}=E_{4}=E_{5} \subseteq E_{6}$.

It remains to show that $E_{1} \neq E_{2} \neq E_{6}$. The latter assertion is easy and we leave it to the reader. As for the former, it is enough to consider the case of a lattice $\Lambda \cong \mathbb{Z}$ of rank 1 . In this case, whenever $p \geq 3$, a non-constant 2-periodic function $f \in E_{1}$ cannot be a trace of a polynomial. In other words, a 2-periodic trace of a polynomial $q \in \mathbb{K}[x]$ on $\mathbb{Z}$ must be constant. Indeed, since $q(1)=q(1-p)=q(0)$ we have $q|2 \mathbb{Z}=q|(2 \mathbb{Z}+1)$. In the remaining case $p=2$, a 3 -periodic function $f$ on $\mathbb{Z}$ with $f(0)=f(1)=0$ and $f(2)=1$ cannot be a trace of a polynomial over $\mathbb{K}$, since $f(0) \neq f(2)$. Thus indeed $E_{1} \neq E_{2}$.

2.6. The function space $\mathcal{F}_{p}(\Lambda, \mathbb{K})$. In positive characteristic, the function space $E_{2}=E_{3}=E_{4}=E_{5}$ of all pluri-periodic functions on $\Lambda$ is not quite appropriate for defining the Fourier transform. More suitable is the translation invariant space

$$
\mathcal{F}_{p}(\Lambda, \mathbb{K})=\operatorname{span}\left(\chi \mid \chi \in \Lambda^{\vee}\right) \subseteq \mathcal{F}
$$

spanned by all characters of $\Lambda$ with values in $\mathbb{K}^{\times}$. Of course, it depends on the characteristic $p$. In Proposition 2.11 below we characterize this subspace in terms of period lattices. To this point we need the following definitions. 
Definition 2.7. Let $\Lambda^{\prime} \subseteq \Lambda$ be a sublattice of finite index. We say that $\Lambda^{\prime}$ is $p$-saturated if $p v \in \Lambda^{\prime}$ implies that $v \in \Lambda^{\prime}$. This holds if and only if the index $\operatorname{ind}_{\Lambda}\left(\Lambda^{\prime}\right)$ is coprime to $p$, or, equivalently, the quotient group $G=\Lambda / \Lambda^{\prime}$ of order $\operatorname{ind}_{\Lambda}\left(\Lambda^{\prime}\right)$ has no $p$-torsion. Clearly, the intersection of two $p$-saturated sublattices is again $p$-saturated. If $\Lambda^{\prime}$ is $p$-saturated then every bigger sublattice $\Lambda^{\prime \prime} \supseteq \Lambda^{\prime}$ is, since $\operatorname{ind}_{\Lambda} \Lambda^{\prime \prime} \mid \operatorname{ind}_{\Lambda} \Lambda^{\prime}$.

If $\Lambda^{\prime}$ is $p$-saturated then the dual group $G^{\vee}$ of $G$ is isomorphic to the group of characters $\operatorname{Char}\left(G, \mathbb{K}^{\times}\right)$. Otherwise $\operatorname{Char}\left(G, \mathbb{K}^{\times}\right) \cong G^{\vee} / G^{\vee}(p)$, where $G^{\vee}(p)$ is the Sylow $p$-component of $G^{\vee}$

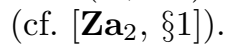

2.8. Every character $\chi \in \Lambda^{\vee}$ regarded as a function in $\mathcal{F}_{p}(\Lambda, \mathbb{K})$ is pluri-periodic, with the period lattice $\Lambda(\chi)=\operatorname{ker}(\chi)$. The quotient group

$$
G(\chi)=\Lambda / \Lambda(\chi) \cong \chi(\Lambda) \subseteq \mathbb{K}^{\times}
$$

is a finite cyclic group of order $\operatorname{ord}(\chi)$ coprime to $p$. Hence the period lattice $\Lambda(\chi)$ is $p$-saturated (see 2.7).

According to 2.6 any function $f \in \mathcal{F}_{p}(\Lambda, \mathbb{K})$ is a linear combination of characters: $f=\sum_{i=1}^{m} \alpha_{i} \chi_{i}$, where $\alpha_{i} \in \mathbb{K}, \chi_{i} \in \Lambda^{\vee}$. Therefore the lattice of periods of $f$,

$$
\Lambda(f) \supseteq \bigcap_{i=1}^{m} \Lambda\left(\chi_{i}\right)
$$

is also $p$-saturated.

Definition 2.9. Let $f \in \mathcal{F}$ be a pluri-periodic function, that is the period lattice $\Lambda(f) \subseteq \Lambda$ has finite index (see Example 2.4(iv)). We say that $f$ is $p$-pure if $\Lambda(f)$ is $p$-saturated. We let $\mathcal{F}_{p p}(\Lambda, \mathbb{K})$ denote the space of all $p$-pure pluri-periodic functions on $\Lambda$ with values in $\mathbb{K}$. This is indeed a linear space because $\Lambda(f+g) \supseteq \Lambda(f) \cap \Lambda(g)$ is $p$-saturated if $\Lambda(f)$ and $\Lambda(g)$ are, see 2.7. Furthermore, the space $\mathcal{F}_{p p}(\Lambda, \mathbb{K})$ is translation invariant, because $\Lambda\left(\tau_{v}(f)\right)=\Lambda(f) \forall v \in \Lambda$. So this is a convolution module over $\mathbb{K}[\Lambda]$.

Remark 2.10. We have shown in Example 2.4 that the trace on $\Lambda$ of a polynomial $q \in \mathbb{K}\left[x_{1}, \ldots, x_{s}\right]$ is $\Lambda^{\prime}$-periodic, where $\Lambda^{\prime}=p \Lambda=\left\langle p v_{1}, \ldots, p v_{s}\right\rangle$. However, the sublattice $\Lambda^{\prime} \subseteq \Lambda$ of finite index is not $p$-saturated. The function $q \mid \Lambda$ is $p$-pure if and only if this is a constant function.

Proposition 2.11. $\mathcal{F}_{p p}(\Lambda, \mathbb{K})=\mathcal{F}_{p}(\Lambda, \mathbb{K})$.

Proof. The inclusion $\mathcal{F}_{p}(\Lambda, \mathbb{K}) \subseteq \mathcal{F}_{p p}(\Lambda, \mathbb{K})$ is immediate from 2.8 and 2.9. It remains to show the converse inclusion. Let $f$ be a pluri-periodic function with period lattice $\Lambda(f) \subseteq \Lambda$ of index coprime to $p$. Then $f=\bar{f} \circ \pi$ is the pullback of a function $\bar{f}: G(f) \rightarrow \mathbb{K}$, where $G(f)=\Lambda / \Lambda(f)$ is a finite abelian group of order coprime with $p$ and $\pi: \Lambda \rightarrow G(f)$ is the canonical surjection. The function space $\mathcal{F}\left(G(f), \mathbb{K}\right.$ ) being spanned by characters of $G(f)$ with values in $\mathbb{K}^{\times}$(see $\mathbf{Z a}_{2}$, Lemma $2.1(\mathrm{a})])$ there is a presentation

$$
\bar{f}=\sum_{i=1}^{n} \alpha_{i} \bar{\chi}_{i} \quad \text { with } \quad \bar{\chi}_{i} \in \operatorname{Char}\left(G(f), \mathbb{K}^{\times}\right) \quad \text { and } \quad \alpha_{i} \in \mathbb{K} .
$$

Thus $f=\sum_{i=1}^{n} \alpha_{i} \chi_{i}$, where the characters $\chi_{i}=\bar{\chi}_{i} \circ \pi \in \Lambda^{\vee}$ are the pullbacks of $\bar{\chi}_{i}, i=1, \ldots, n$. This yields the desired conclusion.

\subsection{Fourier transform.}

2.12. The Fourier transform on $\mathbb{K}[\Lambda]$ sends the group ring $\mathbb{K}[\Lambda]$ isomorphically onto the coordinate ring of the torus $\mathcal{O}\left(\Lambda^{\vee}\right)$. Given a base $\mathcal{V}$ of $\Lambda$ and the identifications $\Lambda \cong \mathbb{Z}^{s}$ and $\Lambda^{\vee} \cong \mathbb{T}^{s}$ as above, 
$F$ is given by

$$
F: f \longmapsto \widehat{f}(z)=\sum_{\lambda \in \mathbb{Z}^{s}} f(\lambda) z^{\lambda}
$$

It sends $\mathbb{K}[\Lambda] \cong \mathbb{K}\left[\mathbb{Z}^{s}\right]$ isomorphically onto the algebra of Laurent polynomials

$$
\mathcal{O}\left(\Lambda^{\vee}\right) \cong \mathcal{O}\left(\mathbb{T}^{s}\right)=\mathbb{K}\left[z, z^{-1}\right]=\mathbb{K}\left[z_{1}, \ldots, z_{n}, z_{1}^{-1}, \ldots, z_{n}^{-1}\right] .
$$

In particular, the delta-function $\delta_{v} \in \mathbb{K}[\Lambda]$ is sent to the Laurent monomial $\widehat{\delta_{v}}=z^{\lambda(v)} \in \mathcal{O}\left(\mathbb{T}^{s}\right)$, which is a character of $\Lambda$. Or, in coordinate-free form, $\widehat{\delta_{v}}=$ eval $_{v}$ regarded as a function on the characters of $\Lambda$. The equality $\delta_{v} * \delta_{w}=\delta_{v+w}$ in $\mathbb{K}[\Lambda]$ amounts to $z^{\lambda(v)} z^{\lambda(w)}=z^{\lambda(v)+\lambda(w)}$ in $\mathcal{O}\left(\mathbb{T}^{s}\right)$. The inverse Fourier transform $F^{-1}: \mathbb{K}\left[z, z^{-1}\right] \rightarrow \mathbb{K}\left[\mathbb{Z}^{s}\right]$ sends a Laurent polynomial $\sum_{\lambda \in \mathbb{Z}^{s}} f(\lambda) z^{\lambda}$ into its coefficient function $f \in \mathbb{K}\left[\mathbb{Z}^{s}\right]$.

2.13. Fourier transform on $\mathcal{F}_{p}(\Lambda, \mathbb{K})$. We let $\mathcal{F}_{\text {fs }}\left(\Lambda^{\vee}, \mathbb{K}\right)$ denote the space of all functions on $\Lambda^{\vee}$ with finite support. It comes equipped with the structure of $\mathcal{O}\left(\Lambda^{\vee}\right)$-module.

Our next goal is to define a Fourier transform on the space $\mathcal{F}_{p}(\Lambda, \mathbb{K}) \subseteq \mathcal{F}$ so to obtain an isomorphism

$$
F: \mathbb{K}[\Lambda] \oplus \mathcal{F}_{p}(\Lambda, \mathbb{K}) \stackrel{\cong}{\longrightarrow} \mathcal{O}\left(\Lambda^{\vee}\right) \oplus \mathcal{F}_{\mathrm{fs}}\left(\Lambda^{\vee}, \mathbb{K}\right)
$$

of $\mathbb{K}[\Lambda]$ - and $\mathcal{O}\left(\Lambda^{\vee}\right)$-modules, respectively. The following observations will be useful in the sequel.

2.14. Suppose that $\Lambda^{\prime}$ is $p$-saturated, and let $\pi: \Lambda \rightarrow G=\Lambda / \Lambda^{\prime}$ be the canonical surjection. The dual group $G^{\vee} \cong \operatorname{Char}\left(G, \mathbb{K}^{\times}\right)$of the finite group $G=\Lambda / \Lambda^{\prime}$ can be realized as the finite subgroup

$$
\Lambda^{\perp}=\left\{\chi \in \Lambda^{\vee} \mid \Lambda^{\prime} \subseteq \operatorname{ker} \chi\right\} \subseteq \Lambda^{\vee}
$$

via the pullback

$$
\pi^{\vee}: G^{\vee} \stackrel{\cong}{\longrightarrow} \Lambda^{\prime \perp}, \quad G^{\vee} \ni \bar{\chi} \longmapsto \chi=\pi^{\vee}(\bar{\chi})=\bar{\chi} \circ \pi \in \Lambda^{\prime \perp} .
$$

Letting $f=\bar{f} \circ \pi \in \mathcal{F}_{\Lambda^{\prime}}(\Lambda, \mathbb{K})$ and $\chi=\bar{\chi} \circ \pi \in \Lambda^{\prime \perp}$ from (1) we obtain a presentation

$$
f=\sum_{\chi \in \Lambda^{\prime \perp}} \alpha(\chi) \cdot \chi
$$

Clearly, every function $f \in \mathcal{F}_{\Lambda^{\prime}}(\Lambda, \mathbb{K})$ admits such a presentation i.e., $\mathcal{F}_{\Lambda^{\prime}}(\Lambda, \mathbb{K})=\operatorname{span}\left(\chi \mid \chi \in \Lambda^{\prime \perp}\right)$.

Definition 2.15. For a function $f \in \mathcal{F}_{p}(\Lambda, \mathbb{K})$ we define its Fourier transform as

$$
F: f=\sum_{i=1}^{m} \alpha_{i} \chi_{i} \longmapsto \widehat{f}=\sum_{i=1}^{m} \alpha_{i} \delta_{\chi_{i}^{-1}}
$$

Since $\operatorname{supp}(\widehat{f}) \subseteq \Lambda(f)^{\perp}$ (see 2.14) we have $\widehat{f} \in \mathcal{F}_{\text {fs }}\left(\Lambda^{\vee}, \mathbb{K}\right)$. Thus the Fourier transform yields an isomorphism

$$
F: \mathcal{F}_{p}(\Lambda, \mathbb{K}) \stackrel{\cong}{\longrightarrow} \mathcal{F}_{\mathrm{fs}}\left(\Lambda^{\vee}, \mathbb{K}\right), \quad \chi \longmapsto \delta_{\chi^{-1}},
$$

with inverse

$$
\mathcal{F}_{\mathrm{fs}}\left(\Lambda^{\vee}, \mathbb{K}\right) \ni \varphi=\sum_{\chi \in \operatorname{supp}(\varphi)} \varphi(\chi) \cdot \delta_{\chi} \longmapsto F^{-1}(\varphi)=\sum_{\chi \in \operatorname{supp}(\varphi)} \varphi(\chi) \cdot \chi^{-1} \in \mathcal{F}_{p}(\Lambda, \mathbb{K}) .
$$

Proposition 2.16. For every $a \in \mathbb{K}[\Lambda]$ and for every $f \in \mathcal{F}_{p}(\Lambda, \mathbb{K})$ we have

$$
\widehat{a * f}=\widehat{a} \cdot \widehat{f} .
$$


Proof. The space $\mathbb{K}[\Lambda]$ being spanned by delta functions and $\mathcal{F}_{p}(\Lambda, \mathbb{K})$ by characters of $\Lambda$, and the Fourier transform being linear, it is enough to verify (4) in the particular case where $a=\delta_{v}$ $(v \in \Lambda)$ and $f=\chi \in \Lambda^{\vee}$. We have

$$
\widehat{\delta_{v} * \chi}=\widehat{\tau_{-v}(\chi)}=\chi^{-1}(v) \cdot \widehat{\chi}=\widehat{\delta_{v}}\left(\chi^{-1}\right) \cdot \delta_{\chi^{-1}}=\widehat{\delta_{v}} \cdot \delta_{\chi^{-1}}=\widehat{\delta_{v}} \cdot \widehat{\chi} .
$$

Indeed, $\widehat{\delta_{v}}=\operatorname{eval}_{v} \in \mathcal{F}\left(\Lambda^{\vee}, \mathbb{K}\right)$. In particular

$$
\chi^{-1}(v)=\operatorname{eval}_{v}\left(\chi^{-1}\right)=\widehat{\delta_{v}}\left(\chi^{-1}\right)
$$

Now (4) follows.

\section{Convolution operators: the scalar case}

\section{1. $\Delta_{a}$-harmonic functions.}

3.1. Let $a \in \mathbb{K}[\Lambda]$. A function $f \in \mathcal{F}$ satisfying the equation

$$
\Delta_{a}(f)=a * f=0
$$

will be called $\Delta_{a}$-harmonic. Choosing a base of $\Lambda$ we identify $\Lambda$ with $\mathbb{Z}^{s}$ and $\Lambda^{\vee}$ with the torus $\mathbb{T}^{s}$. With every convolution operator $\Delta_{a}$ on $\Lambda=\mathbb{Z}^{s}$ we associate its symbolic variety $\Sigma_{a}=\Sigma_{\Delta_{a}}$. This is an affine hypersurface in the dual torus $\Lambda^{\vee} \cong \mathbb{T}^{s}$ defined as

$$
\Sigma_{a}=\left\{z \in \mathbb{T}^{s} \mid \widehat{a}\left(z^{-1}\right)=0\right\},
$$

where $\widehat{a}=F(a)$ is a Laurent polynomial in $z=\left(z_{1}, \ldots, z_{s}\right)$, see $\left[\mathbf{Z \mathbf { a } _ { 2 }}, 2.7\right]$. Every character $\chi \in \Sigma_{a}$ viewed as a function on $\Lambda=\mathbb{Z}^{s}$ is $\Delta_{a}$-harmonic. Indeed, for $z \in \Sigma_{a}$ we have

$$
\Delta_{a}\left(z^{\lambda}\right)=\sum_{v \in \Lambda} a(v) \tau_{-v}\left(z^{\lambda}\right)=\sum_{v \in \Lambda} a(v) z^{\lambda-v}=z^{\lambda} \widehat{a}\left(z^{-1}\right)=0 .
$$

The following proposition is an analog of Theorems 1-3 in $\mathbf{K} \mathbf{u}_{2}$ in the case of scalar functions.

Proposition 3.2. $\quad$ (a) If $a \neq 0$ then a nonzero $\Delta_{a}$-harmonic function on $\Lambda$ cannot have finite support.

(b) Every $\Delta_{a}$-harmonic function $f \in \mathcal{F}_{p}(\Lambda, \mathbb{K})$ is a linear combination of $\Delta_{a}$-harmonic characters $\chi \in \Sigma_{a}$. In other words,

$$
\operatorname{ker}\left(\Delta_{a} \mid \mathcal{F}_{p}(\Lambda, \mathbb{K})\right)=\operatorname{span}\left(\chi \mid \chi \in \Sigma_{a}\right)
$$

Proof. To show (a) suppose that $f \in \mathbb{K}[\Lambda]$ is $\Delta_{a}$-harmonic. Then by Proposition[2.16] $\widehat{a} \cdot \widehat{f}=0$ and so $\widehat{a}, \widehat{f} \in \mathbb{K}\left[z, z^{-1}\right]$ are zero divisors. However, the algebra $\mathbb{K}\left[z, z^{-1}\right]$ of Laurent polynomials being an integral domain this implies that $f=0$. Indeed, $\widehat{a} \neq 0$ by our assumption.

(b) If $a=c \delta_{v}$ i.e., $\Delta_{a}$ is proportional to a shift then the only $\Delta_{a}$-harmonic function in $\mathcal{F}$ is the zero function. Assume further that $a \neq c \delta_{v}$ i.e., $\widehat{a}$ is not a Laurent monomial and so $\Sigma_{a} \neq \emptyset$. Let $f \in \mathcal{F}_{\Lambda^{\prime}}$ be $\Delta_{a}$-harmonic and $\Lambda^{\prime}$-periodic, where $\Lambda^{\prime} \subseteq \Lambda$ is a sublattice of a finite index coprime to $p$, see Proposition 2.11. Then $f$ is the pullback of a function $\bar{f}: G=\Lambda / \Lambda^{\prime} \rightarrow \mathbb{K}$. We let $a_{*}: G \rightarrow \mathbb{K}$ denote the pushforward function defined by

$$
a_{*}\left(v+\Lambda^{\prime}\right)=\sum_{v^{\prime} \in \Lambda^{\prime}} a\left(v+v^{\prime}\right) .
$$

This is a unique function in $\mathcal{F}(G, K)$ satisfying the condition $a_{*} \circ \pi=a * \delta_{\Lambda^{\prime}}$, where $\pi: \Lambda \rightarrow G$ is the canonical surjection and $\delta_{\Lambda^{\prime}}$ stands for the characteristic function of $\Lambda^{\prime} \subseteq \Lambda$ (see $\left[\mathbf{Z} \mathbf{a}_{2}\right.$, (7)]). Since $f$ is $\Delta_{a}$-harmonic, by the Pushforward Lemma 1.1 in $\mathbf{Z a}_{2}, \Delta_{a_{*}}(\bar{f})=0$. It follows that $\bar{f}$ is a linear combination of $a_{*}$-harmonic characters of $G$, see $\mathbf{Z} \mathbf{a}_{2}$, Corollary 2.2]. Their pullbacks are $\Delta_{a}$-harmonic characters of $\Lambda$, and so $f$ is a linear combination of the latter characters, as stated in (b). 
There are the easy equivalencies:

$$
a=0 \quad \Longleftrightarrow \quad \Sigma_{a}=\mathbb{T}^{s} \Longleftrightarrow \operatorname{ker}\left(\Delta_{a} \mid \mathcal{F}_{p}(\Lambda, \mathbb{K})\right)=\mathcal{F}_{p}(\Lambda, \mathbb{K}) .
$$

In the opposite direction, we have the following result (cf. $\left[\mathbf{K \mathbf { u } _ { 1 }}\right.$, Corollary 2]).

Corollary 3.3. There is no nonzero $\Delta_{a}$-harmonic function on $\Lambda$ if and only if $\Sigma_{a}=\emptyset$, if and only if $\Delta_{a}$ is proportional to a shift, if and only if $\Delta_{a}$ is invertible.

3.2. The Floquet-Fermi hypersurfaces. This notion is borrowed in [KuVa, Definition 2].

Definition 3.4. For a convolution operator $\Delta_{a}$, where $a \in \mathbb{K}[\Lambda]$, its Floquet-Fermi hypersurface of level $\mu \in \mathbb{K}$ is

$$
\Sigma_{a, \mu}=\left\{z \in \mathbb{T}^{s} \mid \widehat{a}\left(z^{-1}\right)=\mu\right\}
$$

Actually $\Sigma_{a, \mu}=\Sigma_{a-\mu \delta_{0}}$ is the symbolic hypersurface of the operator $\Delta_{a}-\mu \mathrm{Id}$. We let

$$
E_{a, \mu}=\operatorname{span}\left(\chi \mid \chi \in \Sigma_{a, \mu}\right)
$$

denote the eigenspace of $\Delta_{a} \mid \mathcal{F}_{p}(\Lambda, \mathbb{K})$ with eigenvalue $\mu$.

The following proposition is straightforward. Nevertheless, we provide a short argument.

Proposition 3.5. We have

$$
\mathcal{F}_{p}(\Lambda, \mathbb{K})=\bigoplus_{\mu \in \mathbb{K}} E_{a, \mu}
$$

In particular, the convolution operator $\Delta_{a}$ on the space $\mathcal{F}_{p}(\Lambda, \mathbb{K})$ is diagonalizable in the basis of characters.

Proof. Choosing a base $\left(v_{1}, \ldots, v_{s}\right)$ of the lattice $\Lambda$, we identify $\Lambda$ with $\mathbb{Z}^{s}$ and $\Lambda^{\vee}$ with the torus $\mathbb{T}^{s}$. Letting $\tau_{i}$ denote the shift by $-v_{i}$, the convolution operator $\Delta_{a}$ can be written as a Laurent polynomial in the basic shifts $\tau_{1}, \ldots, \tau_{s}$. Namely,

$$
\Delta_{a}=\sum_{\lambda \in \mathbb{Z}^{s}} a(\lambda) \tau_{-\lambda}=\sum_{\lambda=\left(\lambda_{1}, \ldots, \lambda_{s}\right) \in \mathbb{Z}^{s}} a(\lambda) \prod_{i=1}^{s} \tau_{i}^{\lambda_{i}}=\widehat{a}\left(\tau_{1}, \ldots, \tau_{s}\right) .
$$

Using the equalities $\tau_{i}\left(z^{\lambda}\right)=z_{i}^{-1} \cdot z^{\lambda}$, for every $z \in \mathbb{K}^{\times}$we obtain

$$
\Delta_{a}\left(z^{\lambda}\right)=\widehat{a}\left(\tau_{1}, \ldots, \tau_{s}\right)\left(z^{\lambda}\right)=\widehat{a}\left(z^{-1}\right) \cdot z^{\lambda} .
$$

Now both assertions follow.

Remarks 3.6. 1. Here is an alternative approach. According to Proposition 2.16 the Fourier transform $F$ sends the convolution operator $\Delta_{a} \mid \mathcal{F}_{p}(\Lambda, \mathbb{K})$ to the operator of multiplication by the function $\widehat{a}$ on the space $\mathcal{F}_{\mathrm{fs}}\left(\Lambda^{\vee}, \mathbb{K}\right)=F\left(\mathcal{F}_{p}(\Lambda, \mathbb{K})\right)$. The latter operator being semi-simple, $\Delta_{a}$ is semi-simple too.

2. Let us identify $\Lambda$ with $\mathbb{Z}^{s}$ and $\Lambda^{\vee}$ with the torus $\mathbb{T}^{s}$. Notice that the spectrum of $\Delta_{a} \mid \mathcal{F}_{p}(\Lambda, \mathbb{K})$ coincides with the range of the Laurent polynomial

$$
\widehat{a}\left(z^{-1}\right)=\sum_{\lambda \in \mathbb{Z}^{n}} a(\lambda) z^{-\lambda}
$$

Hence

- $\operatorname{spec}\left(\Delta_{a}\right)=\{\mu\}$ if and only if $a=\mu \delta_{0}$;

- $\operatorname{spec}\left(\Delta_{a}\right)=\mathbb{K}^{\times}$if and only if $a=\alpha \delta_{v}$ for some $\alpha \in \mathbb{K}^{\times}$and some $v \in \Lambda \backslash\{0\}$;

- $\operatorname{spec}\left(\Delta_{a}\right)=\mathbb{K}$ otherwise. 
Furthermore, if $a \neq \alpha \delta_{v}$ then

$$
\begin{aligned}
E_{a, \mu}=\operatorname{span}\left(z^{-\lambda} \mid \widehat{a}(z)=\mu\right)= & \left\{f \in \mathcal{F}_{p}(\Lambda, \mathbb{K}) \mid f(\lambda)=\sum_{z \in \mathbb{T}^{s}} \alpha(z) z^{-\lambda}:\right. \\
& \left.\alpha \in \mathcal{F}_{\mathrm{fs}}\left(\mathbb{T}^{s}, \mathbb{K}\right) \text { s.t. } \operatorname{supp}(\alpha) \subseteq \widehat{a}^{-1}(\mu)\right\} .
\end{aligned}
$$

If $s=\operatorname{rank}(\Lambda)>1$ then every level set $\widehat{a}^{-1}(\mu)$, where $\mu \in \operatorname{spec}\left(\Delta_{a}\right)$, is an infinite countable set (indeed, the algebraically closed field $\mathbb{K}=\overline{\mathbb{F}_{p}}$ is infinite countable). Hence for every $\mu \in \mathbb{K}$ the eigenspace $E_{a, \mu}$ has a countable basis of characters; in particular $\operatorname{dim}\left(E_{a, \mu}\right)=+\infty$. While for $s=1$ all these eigenspaces are finite dimensional.

\section{Convolution operators: the vector case}

Letting $\mathbb{A}^{n}=\mathbb{A}_{\mathbb{K}}^{n}$ be the affine $n$-space over $\mathbb{K}$ and $\left(e_{1}, \ldots, e_{n}\right)$ be the canonical basis of $\mathbb{A}^{n}$, we consider the space $\mathcal{F}^{n}=\mathcal{F}\left(\Lambda, \mathbb{A}^{n}\right)$ of all $\mathbb{K}$-valued vector functions on $\Lambda$, and its subspaces $\mathbb{K}[\Lambda]^{n}$ of all vector functions on $\Lambda$ with finite support and $\mathcal{F}_{p}\left(\Lambda, \mathbb{A}^{n}\right)$ of all $p$-pure pluri-periodic vector functions on $\Lambda$. The vector functions on $\Lambda=\mathbb{Z}^{s}$ of the form $f(\lambda)=\chi \cdot u=z^{\lambda} \cdot u$, where $z \in \mathbb{T}^{s}=\Lambda^{\vee}$ and $u \in \mathbb{A}^{n}$, will be called elementary. These are analogs in positive characteristic of the classical Bloch functions. Thus any $f \in \mathcal{F}_{p}\left(\Lambda, \mathbb{A}^{n}\right)$ is a linear combination of elementary functions.

4.1. Identifying as before $\Lambda$ with $\mathbb{Z}^{s}$ and $\Lambda^{\vee}$ with $\mathbb{T}^{s}$, the Fourier transform sends a vector function $f \in \mathbb{K}[\Lambda]^{n}$ into the vector Laurent polynomial

$$
\widehat{f}(z)=\sum_{\lambda \in \mathbb{Z}^{n}} f(\lambda) z^{\lambda}, \quad z \in \mathbb{T}^{s}
$$

where $f(\lambda) \in \mathbb{A}^{n} \forall \lambda \in \mathbb{Z}^{s}$. In particular, a basic vector delta-function $\delta_{i, \lambda}=\delta_{\lambda} \cdot e_{i} \in \mathbb{K}[\Lambda]^{n}$, where $\lambda \in \Lambda=\mathbb{Z}^{s}$, is sent to the vector Laurent monomial $\widehat{\delta_{i, \lambda}}=z^{\lambda} \cdot e_{i}(i=1, \ldots, n)$. A pluri-periodic vector function

$$
f=\sum_{i=1}^{m} \alpha_{i} \chi_{i} \in \mathcal{F}_{p}\left(\Lambda, \mathbb{A}^{n}\right),
$$

where $\alpha_{i} \in \mathbb{A}^{n}$ and $\chi_{i} \in \Lambda^{\vee}$, is sent to the vector function on $\Lambda^{\vee}$ with finite support

$$
\widehat{f}=\sum_{i=1}^{m} \alpha_{i} \delta_{\chi_{i}^{-1}} \in \mathcal{F}_{\mathrm{fs}}\left(\Lambda^{\vee}, \mathbb{A}^{n}\right) .
$$

4.1. $\Delta$-harmonic vector functions. Consider an endomorphism $\Delta \in \operatorname{End}\left(\mathbb{K}[\Lambda]^{n}\right)$ commuting with shifts on $\Lambda$. Letting

$$
\Delta\left(\delta_{i, 0}\right)=a_{i}=\left(a_{i 1}, \ldots, a_{i n}\right) \in \mathbb{K}[\Lambda]^{n}, \quad i=1, \ldots, n,
$$

we get a square matrix $A=\left(a_{i j}\right)$ of order $n$ with entries in $\mathbb{K}[\Lambda]$. The images of the remaining basic vector functions $\delta_{i, v}$ can be recovered as suitable shifts of the vectors $a_{i}$ :

$$
\Delta\left(\delta_{i, v}\right)=\Delta\left(\tau_{-v} \delta_{i, 0}\right)=\tau_{-v}\left(\Delta\left(\delta_{i, 0}\right)\right)=\tau_{-v}\left(a_{i}\right) .
$$

If $f=\left(f_{1}, \ldots, f_{n}\right) \in \mathbb{K}[\Lambda]^{n}$ and $g=\Delta(f)=\left(g_{1}, \ldots, g_{n}\right) \in \mathbb{K}[\Lambda]^{n}$ then $g=A * f$ i.e.,

$$
g_{i}=\sum_{i=1}^{n} a_{i j} * f_{j}, \quad i=1, \ldots, n
$$

In other words, $\Delta$ acts on $\mathbb{K}[\Lambda]^{n}$ as a square matrix of convolution operators $\Delta_{A}=\left(\Delta_{a_{i j}}\right)$. Such operator $\Delta$ can be applied to an arbitrary vector function $f=\left(f_{1}, \ldots, f_{n}\right) \in \mathcal{F}\left(\Lambda, \mathbb{A}^{n}\right)=\mathcal{F}^{n}$. This yields an extension of $\Delta$ to $\mathcal{F}^{n}$, which we denote by the same letter. The extended endomorphism $\Delta \in \operatorname{End}\left(\mathcal{F}^{n}\right)$ leaves invariant any translation invariant subspace. In particular, it acts on the subspace $\mathcal{F}_{p}\left(\Lambda, \mathbb{A}^{n}\right) \subseteq \mathcal{F}^{n}$. 
The Fourier transform sends $A$ into a matrix of Laurent polynomials $\widehat{A}=\left(\widehat{a}_{i j}\right)$. By Proposition 2.16 the relation $g=A * f$, where $f, g \in \mathcal{F}_{p}\left(\Lambda, \mathbb{A}^{n}\right)$, is transformed into

$$
\widehat{g}=\widehat{A} \cdot \widehat{f},
$$

where $\widehat{f}, \widehat{g} \in \mathcal{F}_{\mathrm{fs}}\left(\Lambda^{\vee}, \mathbb{A}^{n}\right)$.

The following is an analog of Theorems 1-3 in $\mathbf{K} \mathbf{u}_{1}$ for vector functions, with a similar proof (cf. also Theorem 8 in $\mathbf{K u}_{2}$ ).

Theorem 4.2. Let $\Delta \in \operatorname{End}\left(\mathbb{K}[\Lambda]^{n}\right)$ be an endomorphism commuting with shifts. Consider the equation

$$
\Delta(f)=0
$$

where $\Delta$ acts on $\mathcal{F}^{n}$ as in (마). Then the following hold.

(a) There exists a non-zero solution of (7) with finite support $f \in \mathbb{K}[\Lambda]^{n}$ if and only if $\operatorname{det} \widehat{A}=0$.

(b) Let $\Lambda^{\prime} \subseteq \Lambda$ be a sublattice of finite index coprime to $p$. There exists a $\Lambda^{\prime}$-periodic solution $f \in \mathcal{F}_{\Lambda^{\prime}}\left(\Lambda, \mathbb{A}^{n}\right)$ of (7) if and only if there exists a character $\chi \in \Lambda^{\prime \perp}$ with $\operatorname{det} \widehat{A}\left(\chi^{-1}\right)=0$. Every such solution is a linear combination of elementary solutions

$$
f=\sum_{i=1}^{m} \chi_{i} \cdot u_{i}
$$

where $\chi_{i} \in \Lambda^{\prime \perp}$ with $\operatorname{det} \widehat{A}\left(\chi_{i}^{-1}\right)=0$ and $u_{i} \in \operatorname{ker}\left(\widehat{A}\left(\chi_{i}^{-1}\right)\right) \forall i=1, \ldots, m$. Vice versa, any vector function as in (8) is a $\Lambda^{\prime}$-periodic solution of (7).

Proof. (a) Choosing a base of $\Lambda$ we identify $\Lambda$ with $\mathbb{Z}^{s}$ and $\Lambda^{\vee}$ with $\mathbb{T}^{s}$. Applying the Fourier transform to (7) yields the equation

$$
\widehat{A} \cdot \widehat{f}=0 \text {. }
$$

We can treat (9) as a linear system over the rational function field $\mathbb{K}(z)$. By the usual linear algebra argument, this system admits a rational solution $\widehat{f} \in[\mathbb{K}(z)]^{n}=\left[\mathbb{K}\left(z_{1}, \ldots, z_{s}\right)\right]^{n}$ if and only if $\operatorname{det} \widehat{A}=0$. Multiplying such a solution $\widehat{f}$ by a suitable polynomial $q \in \mathbb{K}[z]$ we obtain a polynomial solution of (9), hence a solution in vector Laurent polynomials. In turn, (7) admits a solution with finite support.

(b) If $f=\left(f_{1}, \ldots, f_{n}\right)$ is a nonzero $\Lambda^{\prime}$-periodic solution of (77) then $\widehat{f}=\left(\widehat{f}_{1}, \ldots, \widehat{f_{n}}\right)$ is a nonzero solution of (9) supported on $\Lambda^{\prime \perp}$. Hence $\widehat{f}$ does not vanish at some point $\chi \in \Lambda^{\prime \perp}$ and so $(\operatorname{det} \widehat{A})(\chi)=$ 0 . However, any vector $u \in \operatorname{ker}\left(\widehat{A}\left(\chi^{-1}\right)\right)$ yields an elementary solution $f=\chi \cdot u$ of (7). Now the first assertion in (b) follows. The last assertion of (b) is evident.

To show the second assertion in (b), let us consider the finite abelian group $G=\Lambda / \Lambda^{\prime}$ of order coprime to $p$. Every $\Lambda^{\prime}$-periodic vector function $f \in \mathcal{F}_{\Lambda^{\prime}}\left(\Lambda, \mathbb{A}^{n}\right)$ is pullback of a vector function $\bar{f} \in$ $\mathcal{F}\left(G, \mathbb{A}^{n}\right)$. The image $g=A * f$ being also $\Lambda^{\prime}$-periodic, it is pullback of a function $\bar{g} \in \mathcal{F}\left(G, \mathbb{A}^{n}\right)$. The endomorphism $\Delta_{*}: \bar{f} \longmapsto \bar{g}$ is given by the pushforward matrix $A_{*}=\left(a_{i j *}\right)$, where $a_{i j *} \in \mathcal{F}(G, \mathbb{K})$ $\forall(i, j)$. The latter acts via

$$
\bar{g}_{i}=\sum_{j=1}^{n} a_{i j *} * \bar{f}_{j}, \quad i=1, \ldots, n
$$

(cf. the Pushforward Lemma 1.1 in $\mathbf{Z a}_{2}$ ). Since $p \nmid \operatorname{ord} G$ the Fourier transform is well defined on the space $\mathcal{F}(G, \mathbb{K})$ (cf. $\left.\mathbf{Z a}_{2}\right)$. Applying the Fourier transform on $G$ yields a matrix function 
$\widehat{A_{*}}=\left(\widehat{a_{i j *}}\right)$ on the dual group $G^{\vee}$ which acts on vector functions on $G^{\vee}$ via the usual matrix multiplication. Thus (77) leads to equation

$$
\widehat{A_{*}} \cdot \widehat{\bar{f}}=0 \text {. }
$$

Any solution $f$ of (7) gives a solution $\widehat{\bar{f}}$ of (10). Writing this solution as a linear combination of delta-functions yields a presentation of $\bar{f}$ as a linear combination of elementary solutions on $G$. The pullback of this presentation gives (8).

Definition 4.3. Consider as before an endomorphism $\Delta \in \operatorname{End}\left(\mathbb{K}[\Lambda]^{n}\right)$ commuting with shifts, where $n \in \mathbb{N}$. Similarly to the scalar case (where $n=1$ ) we call the hypersurface

$$
\Sigma_{\Delta}=\left\{z \in \Lambda^{\vee} \mid \operatorname{det} \widehat{A}\left(z^{-1}\right)=0\right\}
$$

the symbolic variety of $\Delta$ (cf. 3.1). A point $z \in \Sigma_{\Delta}$ corresponding to a character $\chi=z^{\lambda}$ of $\Lambda \cong \mathbb{Z}^{s}$ is called a multiplier of (7). In case (b) of Theorem 4.2 the multipliers run over the intersection $\Sigma_{\Delta} \cap \Lambda^{\prime \perp}$. Thus every $\Lambda^{\prime}$-periodic solution $f$ of (7) as in (8) can be written as

$$
f(\lambda)=\sum_{z \in \Sigma_{\Delta} \cap \Lambda^{\prime} \perp} z^{\lambda} \cdot u(z)
$$

Corollary 4.4. Under the assumptions of Theorem 4.2 the equation (7) admits a nonzero solution $f \in \mathcal{F}_{p}(\Lambda, \mathbb{K})$ if and only if it admits such an elementary solution, if and only if $\Sigma_{\Delta} \neq \emptyset$. Moreover,

$$
\operatorname{ker}(\Delta)=\operatorname{span}\left(z^{\lambda} \cdot u \mid z \in \Sigma_{\Delta}, u \in \operatorname{ker} \widehat{A}\left(z^{-1}\right)\right) .
$$

Remark 4.5. The finite subgroups $\Lambda^{\prime}$ exhaust the dual torus $\Lambda^{\vee} \cong \mathbb{T}^{s}$ when $\Lambda^{\prime}$ runs over all sublattices of $\Lambda$ of finite indices coprime to $p$. Given $\Delta \in \operatorname{End} \mathbb{K}[\Lambda]^{n}$ commuting with shifts, it would be interesting to find the counting function $\operatorname{card}\left(\Sigma_{\Delta} \cap \Lambda^{\prime}{ }^{\perp}\right)$ as a suitable analog of the Weil zeta-function.

4.2. The Jordan form of a matrix convolution operator. Over an algebraically closed field, every square matrix admits a Jordan basis [Bou, Ch. VII, §5.2, Prop. 5]. We construct below such a basis for any matrix convolution operator.

Definition 4.6. Given an operator $\Delta \in \operatorname{End}(\mathbb{K}[\Lambda])$ commuting with shifts, for every $\mu \in \mathbb{K}$ we consider its Floquet-Fermi hypersurface

$$
\Sigma_{\Delta, \mu}=\left\{z \in \mathbb{T}^{s} \mid \operatorname{det}\left(\widehat{A}\left(z^{-1}\right)-\mu \cdot I_{n}\right)=0\right\}
$$

the corresponding eigenspace

$$
E_{\Delta, \mu}=\operatorname{ker}\left((\Delta-\mu \cdot \operatorname{Id}) \mid \mathcal{F}_{p}(\Lambda, \mathbb{K})\right)
$$

and the generalized eigenspace

$$
E_{\Delta}^{(\mu)}=\operatorname{ker}\left((\Delta-\mu \cdot \operatorname{Id})^{n} \mid \mathcal{F}_{p}(\Lambda, \mathbb{K})\right)
$$

The proof of the following theorem is straightforward.

Theorem 4.7. Let $\Delta \in \operatorname{End}(\mathbb{K}[\Lambda])$ be an endomorphism commuting with shifts, extended via (6) to the space $\mathcal{F}_{p}(\Lambda, \mathbb{K})$ as a matrix convolution operator. Then the following hold.

(a) There is a decomposition

$$
\mathcal{F}_{p}\left(\Lambda, \mathbb{A}^{n}\right)=\bigoplus_{\mu \in \mathbb{K}} E_{\Delta}^{(\mu)}
$$


(b) Every generalized eigenspace $E_{\Delta}^{(\mu)} \subseteq \mathcal{F}_{p}(\Lambda, \mathbb{K})$ is generated by elementary vector-functions. Namely,

$$
\begin{gathered}
E_{\Delta}^{(\mu)}=\operatorname{span}\left(z^{\lambda} \cdot u \mid z \in \Sigma_{\Delta, \mu}, u \in \operatorname{ker}\left(A\left(z^{-1}\right)-\mu \cdot I_{n}\right)^{n}\right) \\
=\left\{f \in \mathcal{F}_{p}\left(\Lambda, \mathbb{A}^{n}\right) \mid f(\lambda)=\sum_{z \in \Sigma_{a, \mu}} \alpha(z) z^{\lambda}, \quad \text { where } \alpha \in \mathcal{F}_{\mathrm{fs}}\left(\Lambda^{\vee}, \mathbb{A}^{n}\right)\right. \text { is s.t. } \\
\left.\alpha(z) \in \operatorname{ker}\left(\widehat{A}\left(z^{-1}\right)-\mu \cdot I_{n}\right)^{n} \forall z \in \operatorname{supp}(\alpha)\right\} .
\end{gathered}
$$

(c) The induced endomorphism $\Delta \in \operatorname{End}\left(\mathcal{F}_{p}(\Lambda, \mathbb{K})\right)$ admits a Jordan basis consisting of elementary vector functions. More precisely, fixing for every $z \in \mathbb{T}^{n}$ a Jordan basis $\left(u_{1}(z), \ldots, u_{n}(z)\right)$ for $\widehat{A}\left(z^{-1}\right)$ in $\mathbb{A}^{n}$ we obtain a Jordan basis $\left(z^{\lambda} \cdot u_{i}(z) \mid z \in \mathbb{T}^{n}\right)$ for $\Delta$ in $\mathcal{F}_{p}\left(\Lambda, \mathbb{A}^{n}\right)$.

Remark 4.8. À priori, the choice of the vector functions $u_{i}(z)$ in (c) does not suppose any specific dependence on $z=\left(z_{1}, \ldots, z_{s}\right)$. However, they can be chosen as (non-rational, in general) algebraic vector functions in $z$.

4.3. Convolution equations over finite fields. Taking suitable traces we can obtain solutions of the linear equation (7) with values in a finite field. We discuss below this matter in more detail.

Given a square matrix function $A=\left(a_{i j}\right)$ of order $n$ with entries $a_{i j} \in \mathbb{K}[\Lambda]$, we consider as before the matrix convolution equation

$$
A * f=0, \quad \text { where } f=\left(f_{1}, \ldots, f_{n}\right) \in \mathcal{F}_{p}\left(\Lambda, \mathbb{A}^{n}\right) .
$$

The proof of the following proposition follows the lines of the proof of Theorem 4.4 in $\mathbf{Z a}_{2}$.

Proposition 4.9. Consider a finite Galois field $G F(q)=\mathbb{F}_{q} \subset \mathbb{K}$, where $q=p^{\sigma}$. Suppose that $a_{i j} \in G F(q)[\Lambda] \forall i, j=1, \ldots, n$. If (11) admits a nonzero solution $f \in \mathcal{F}_{p}\left(\Lambda, \mathbb{A}^{n}\right)$, then it admits also a nonzero solution $\tilde{f} \in \mathcal{F}_{p}\left(\Lambda, \mathbb{A}^{n}\right)$ with values in the field $G F(q)$.

Proof. The equation (11) is equivalent to the system

$$
\sum_{j=1}^{n} a_{i j} * f_{j}=0, \quad i=1, \ldots, n .
$$

Since $G F(q)$ is the subfield of invariants of the Frobenius automorphism $z \longmapsto z^{q}$ acting on $\mathbb{K}$, applying this automorphism yields the equalities

$$
\sum_{j=1}^{n} a_{i j} * f_{j}^{q}=0, \quad i=1, \ldots, n .
$$

That is $f^{q}=\left(f_{1}^{q}, \ldots, f_{n}^{q}\right) \in \mathcal{F}_{p}\left(\Lambda, \mathbb{A}^{n}\right)$ is again a solution of (11).

The function $f_{j}$ being pluri-periodic its image $f_{j}(\Lambda) \subseteq \mathbb{K}$ is finite. Let $q(f)=p^{r(f)}$ be such that $\mathrm{GF}(q(f))$ is the smallest subfield of $\mathbb{K}$ containing $\mathrm{GF}(q)$ and all the images $f_{j}(\Lambda), j=1, \ldots, n$. We let $\operatorname{Tr}(f)=\left(\operatorname{Tr}\left(f_{1}\right), \ldots, \operatorname{Tr}\left(f_{n}\right)\right)$, where

$$
\operatorname{Tr}\left(f_{j}\right)=\operatorname{Tr}_{G F(q(f)): G F(q)}\left(f_{j}\right):=f_{j}+f_{j}^{q}+\ldots+f_{j}^{q^{r(f)-1}} \in \mathcal{F}_{p}(G, \mathbb{K}) .
$$

The trace $\operatorname{Tr}(f)$ is fixed by the Frobenius automorphism, hence it represents a solution of (11) with values in the finite field $G F(q)$. It remains to show that such a solution can be chosen nonzero.

Let $\Delta \in \operatorname{End}\left(\mathcal{F}_{p}\left(\Lambda, \mathbb{A}^{n}\right)\right)$ denote the matrix convolution operator $\Delta(f)=A * f$. By Corollary $4.4 \operatorname{ker}(\Delta)$ is spanned by elementary solutions $f=z^{\lambda} \cdot u$, where $z \in \Sigma_{\Delta} \subseteq \mathbb{T}^{s}$. By our assumption $\operatorname{ker}(\Delta) \neq(0)$, hence $\Sigma_{\Delta} \neq \emptyset$. Choosing $z \in \Sigma_{\Delta}$ we let

$$
r(z)=\min \left\{r \mid z^{\lambda},\left(z^{q}\right)^{\lambda}, \ldots,\left(z^{q^{r-1}}\right)^{\lambda} \quad \text { are all distinct }\right\} .
$$


The entries of the matrix $\widehat{A}\left(z^{-1}\right)$ being Laurent polynomials with coefficients in $\operatorname{GF}(q)$, there exists a vector $u \in \operatorname{ker}\left(\widehat{A}\left(z^{-1}\right)\right), u \neq 0$, with coordinates in the field $\operatorname{GF}\left(q^{r(z)}\right)$. Thus $f=z^{\lambda} \cdot u$ is a nonzero elementary solution with values in the field $G F\left(q^{r(z)}\right)$. Let $j \in\{1, \ldots, n\}$ be such that $u_{j} \neq 0$. Then $f_{j}=u_{j} \cdot z^{\lambda} \neq 0$. Moreover,

$$
\operatorname{Tr}\left(f_{j}\right)=u_{j} \cdot z^{\lambda}+u_{j}^{q} \cdot\left(z^{q}\right)^{\lambda}+\ldots+u_{j}^{q^{r(f)-1}} \cdot\left(z^{q^{r(f)-1}}\right)^{\lambda}
$$

is a non-trivial linear combination of pairwise distinct characters. Therefore $\operatorname{Tr}(f)$ is a nonzero solution with values in the field $G F(q)$, as required.

Remark 4.10. By Theorem 4.4 in $\left[\mathbf{Z a}_{2}\right.$, in the scalar case the subspace $\operatorname{ker}(\Delta) \cap \mathcal{F}_{p}(\Lambda, \operatorname{GF}(q))$ is spanned by suitable traces of characters (i.e., of elementary solutions).

\section{Examples and applications}

The constructions in 5.15 .2 below are borrowed from $\mathbf{K \mathbf { u } _ { 1 }}$.

5.1. Let $\Lambda=\mathbb{Z}^{s}$ be a free abelian group acting on a countable set $V$ with a finite quotient $V / \Lambda$ of cardinality $n$. Choosing a fundamental domain $\Omega=\left\{v_{1}, \ldots, v_{n}\right\} \subseteq V$ (i.e., a set of representatives of the orbits of $\Lambda$ ) we obtain a disjoint partition

$$
V=\bigsqcup_{v \in \Lambda} \tau_{v}(\Omega)
$$

According to this partition, every function $f \in \mathcal{F}(V, \mathbb{K})$ yields a vector function fragm $(f) \in \mathcal{F}\left(\Lambda, \mathbb{A}_{\mathbb{K}}^{n}\right)$. This provides an isomorphism of 'fragmentation'

$$
\text { fragm : } \mathcal{F}(V, \mathbb{K}) \stackrel{\cong}{\longrightarrow} \mathcal{F}\left(\Lambda, \mathbb{A}^{n}\right) \text {. }
$$

Any endomorphism $\Delta \in$ End $(\mathcal{F}(V, \mathbb{K}))$ commuting with the $\Lambda$-action on the space $\mathcal{F}(V, \mathbb{K})$ amounts to an endomorphism $\operatorname{Fragm}(\Delta) \in \operatorname{End}\left(\mathcal{F}\left(\Lambda, \mathbb{A}^{n}\right)\right)$ commuting with shifts. This yields a representation

$$
\operatorname{Fragm}: \operatorname{End}_{\Lambda}(\mathcal{F}(V, \mathbb{K})) \rightarrow \operatorname{End}_{\Lambda}\left(\mathcal{F}\left(\Lambda, \mathbb{A}^{n}\right)\right),
$$

where $\operatorname{End}_{\Lambda}$ stands for the set of endomorphisms commuting with the action of $\Lambda$. In 5.2 and 5.3 below we provide two particular occasions of this construction.

5.2. Covering graphs. Let $\Gamma$ be a finite connected graph with $n$ vertices. Consider the maximal abelian covering $\widetilde{\Gamma} \rightarrow \Gamma$, which corresponds to the commutator subgroup of the fundamental group $\pi_{1}(\Gamma)$. The deck transformation group (i.e., the Galois group) of the covering $\widetilde{\Gamma} \rightarrow \Gamma$ is a free abelian group $H_{1}(\Gamma ; \mathbb{Z}) \cong \mathbb{Z}^{s}$, where $s=b_{1}(\Gamma)$. It acts freely on $\tilde{\Gamma}$ with quotient $\Gamma=\widetilde{\Gamma} / \mathbb{Z}^{s}$. Let $V$ be the set of vertices of the periodic graph $\widetilde{\Gamma}$. Every convolution operator $\Delta \in \operatorname{End}(\mathcal{F}(V, \mathbb{K}))$ gives rise to an operator $\operatorname{Fragm}(\Delta) \in \operatorname{End}_{\Lambda}\left(\mathcal{F}\left(\mathbb{Z}^{s}, \mathbb{A}^{n}\right)\right)$ commuting with shifts. In particular, this applies to the Markov and the Laplace operators on $\widetilde{\Gamma}$ properly adopted to the case of positive characteristic. As before, this yields a representation of the algebra of convolution operators on $\tilde{\Gamma}$. The same construction works for any abelian covering of $\Gamma$.

To give further examples, let us consider the following toy model.

5.3. Fragmentation: a toy model. Let $\Lambda$ be a sublattice of finite index $n$ in a bigger lattice $\widetilde{\Lambda}$. Consider the action of $\Lambda$ on the set $V=\widetilde{\Lambda}$ by shifts. Then any endomorphism $\Delta \in \operatorname{End}_{\Lambda}(\mathcal{F}(\widetilde{\Lambda}, \mathbb{K}))$ commuting with shifts by $\Lambda$ gives rise to an endomorphism $\operatorname{Fragm}_{\Lambda}(\Delta) \in \operatorname{End}_{\Lambda}\left(\mathcal{F}\left(\Lambda, \mathbb{A}^{n}\right)\right)$ commuting with shifts. In particular, for different choices of $\Lambda$ this yields a collection of representations

$$
\operatorname{Fragm}_{\Lambda}: \mathbb{K}[\widetilde{\Lambda}] \rightarrow \operatorname{End}_{\Lambda}\left(\mathcal{F}\left(\Lambda, \mathbb{A}^{n}\right)\right)
$$

of the convolution algebra on $\widetilde{\Lambda}$. 
New representations appear when restricting the morphism of fragmentation to a translation invariant subspace e.g., to the subspace $\mathcal{F}_{p}\left(\Lambda, \mathbb{A}^{n}\right) \subseteq \mathcal{F}\left(\Lambda, \mathbb{A}^{n}\right)$. We need the following simple lemma.

Lemma 5.4. In the notation as before, for any $n$ coprime to $p$ we have

$$
\operatorname{fragm}_{\Lambda}: \mathcal{F}_{p}(\widetilde{\Lambda}, \mathbb{K}) \stackrel{\cong}{\longrightarrow} \mathcal{F}_{p}\left(\Lambda, \mathbb{A}^{n}\right)
$$

Proof. For every $f=\left(f_{1}, \ldots, f_{n}\right) \in \mathcal{F}_{p}(\Lambda, \mathbb{K})^{n}$, the finite index sublattice of $\Lambda$,

$$
\bigcap_{i=1}^{n} \Lambda\left(f_{i}\right) \subseteq \Lambda(f)
$$

is $p$-saturated. Therefore by $2.7 \Lambda(f)$ is $p$-saturated as well. This gives the inclusion $\mathcal{F}_{p}(\Lambda, \mathbb{K})^{n} \subseteq$ $\mathcal{F}_{p}\left(\Lambda, \mathbb{A}^{n}\right)$. The opposite inclusion is evident. Now the last equality in (12) follows.

Letting furthermore $f=\operatorname{fragm}_{\Lambda}(\tilde{f})$, where $\tilde{f} \in \mathcal{F}_{p}(\widetilde{\Lambda}, \mathbb{K})$, it is easily seen that the sublattice of finite index

$$
\Lambda(f) \supseteq \Lambda \cap \widetilde{\Lambda}(\tilde{f})
$$

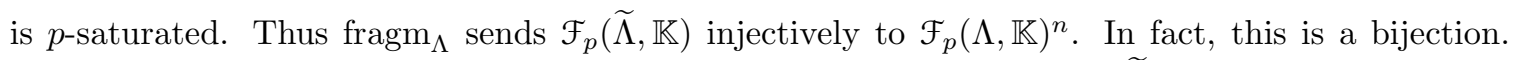
Indeed, let $\left(v_{1}, \ldots, v_{n}\right)$ be a set of representatives of the cosets of $\Lambda$ in $\widetilde{\Lambda}$. For a vector function $f=\left(f_{1}, \ldots, f_{n}\right) \in \mathcal{F}_{p}(\Lambda, \mathbb{K})^{n}$ we let

$$
\tilde{f}(v)=f_{i}\left(v-v_{i}\right) \quad \text { if } \quad v-v_{i} \in \Lambda .
$$

It is easily seen that $f=\operatorname{fragm}_{\Lambda}(\tilde{f})$. Moreover, $\widetilde{\Lambda}(\tilde{f}) \supseteq \Lambda(f)$, where $\Lambda(f)$ is $p$-saturated in $\widetilde{\Lambda}$. Hence $\widetilde{\Lambda}(\tilde{f})$ is also $p$-saturated and so fragm $_{\Lambda}$ in (12) is an isomorphism.

In 5.5,5.8 below we apply our toy model 5.3 in different concrete settings.

Example 5.5. Consider a lattice $\widetilde{\Lambda}=\mathbb{Z}$ of $\operatorname{rank} s=1$ and the shift $\tau=\tau_{-1}$ on $\widetilde{\Lambda}$. By Proposition 3.5 the induced action of $\tau$ on the space $\mathcal{F}_{p}(\widetilde{\Lambda}, \mathbb{K})=\operatorname{span}\left(z^{\lambda} \mid z \in \mathbb{K}^{\times}\right)$can be diagonalized in the basis of characters. More precisely, $\tau\left(z^{\lambda}\right)=z^{-1} \cdot z^{\lambda}$, so $\operatorname{spec}(\tau)=\mathbb{K}^{\times}$and

$$
\mathcal{F}_{p}(\widetilde{\Lambda}, \mathbb{K})=\bigoplus_{\mu \in \mathbb{K}^{\times}} E_{\tau, \mu}, \quad \text { where } \quad E_{\tau, \mu}=\mathbb{K} \cdot \mu^{-\lambda}
$$

The same is true for any convolution operator $\Delta_{a}$ on $\widetilde{\Lambda}$, where $a \in \mathbb{K}[\widetilde{\Lambda}]$. Indeed, we have $\Delta_{a}=\widehat{a}(\tau)$ and so

$$
\Delta_{a}\left(z^{\lambda}\right)=\widehat{a}\left(z^{-1}\right) \cdot z^{\lambda} \quad \forall z \in \mathbb{K}^{\times} .
$$

Consider further a sublattice $\Lambda=n \mathbb{Z}$ of $\widetilde{\Lambda}=\mathbb{Z}$ and the fragmentation $\operatorname{Fragm}(\tau)=\operatorname{Fragm}_{n}(\tau) \in$ $\operatorname{End}_{\Lambda}\left(\mathcal{F}\left(\Lambda, \mathbb{A}^{n}\right)\right)$. Since $\tau\left(\delta_{k}\right)=\delta_{k+1} \forall k \in \mathbb{Z}=\widetilde{\Lambda}$, for the basic vector delta-functions $\delta_{i, \lambda}=\delta_{\lambda} \cdot e_{i}$ on $\Lambda$ we have

$$
\operatorname{Fragm}(\tau)\left(\delta_{0, k}\right)=\delta_{0, k+1} \text { for } k=0, \ldots, n-2, \text { and } \operatorname{Fragm}(\tau)\left(\delta_{0, n-1}\right)=\delta_{1,0} .
$$

The corresponding matrix of Laurent polynomials $\widehat{A}(z)=\widehat{A_{\operatorname{Fragm}(\tau)}}(z)$ and its inverse matrix $\widehat{A}(z)^{-1}=\widehat{A_{\operatorname{Fragm}\left(\tau^{-1}\right)}}(z)$ are

$$
\widehat{A}(z)=\left(\begin{array}{ccccc}
0 & 0 & \ldots & 0 & z \\
1 & 0 & \ldots & 0 & 0 \\
0 & 1 & \ldots & 0 & 0 \\
\vdots & \ddots & \ddots & \ddots & \vdots \\
0 & 0 & \ldots & 1 & 0
\end{array}\right) \quad \operatorname{resp} . \widehat{A}(z)^{-1}=\left(\begin{array}{ccccc}
0 & 1 & 0 & \ldots & 0 \\
0 & 0 & 1 & \ldots & 0 \\
\vdots & \ddots & \ddots & \ddots & \vdots \\
0 & 0 & \ldots & 0 & 1 \\
z^{-1} & 0 & \ldots & 0 & 0
\end{array}\right)=\widehat{A}^{t}\left(z^{-1}\right) .
$$


Thus

where

$$
\operatorname{det}\left(x I_{n}-\widehat{A}(z)\right)=x^{n}-z=\prod_{i=1}^{n}\left(x-\xi_{i}\right)
$$

$$
\left\{\xi_{1}, \ldots, \xi_{n}\right\}=\{\sqrt[n]{z}\}=\operatorname{spec}(\widehat{A}(z)) \subseteq \mathbb{K}^{\times} .
$$

Suppose that $p \nmid n$. Then for $z \in \mathbb{K}^{\times}$we have $\xi_{i} \neq \xi_{j}$ if $i \neq j$. So the matrix $\widehat{A}\left(z^{-1}\right)$ with eigenvalues $\xi_{1}^{-1}, \ldots, \xi_{n}^{-1}$ can be diagonalized in the basis of eigenvectors

$$
v_{i}=\left(\begin{array}{c}
1 \\
\xi_{i} \\
\vdots \\
\xi_{i}^{n-1}
\end{array}\right), \quad i=1, \ldots, n
$$

where

$$
\widehat{A}\left(z^{-1}\right)\left(v_{i}\right)=\xi_{i}^{-1} v_{i} \quad \forall i=1, \ldots, n .
$$

The collection of elementary vector functions

$$
\left(\lambda \longmapsto v_{i} \cdot z^{\lambda} \mid z \in \mathbb{K}^{\times}, i=1, \ldots, n\right)
$$

form a diagonalizing basis for the endomorphism $\operatorname{Fragm}_{n}(\tau) \in \operatorname{End}_{\Lambda}\left(\mathcal{F}_{p}\left(\Lambda, \mathbb{A}^{n}\right)\right)(\operatorname{cf}$. Proposition 4.7(c)). Actually $v_{i} \cdot z^{\lambda}=\operatorname{fragm}_{n}\left(\xi_{i}^{\lambda}\right)$, where $\lambda \longmapsto \xi_{i}^{\lambda}$ is a character of $\tilde{\Lambda}=\mathbb{Z}$.

In conclusion, the isomorphism of fragmentation fragm $_{n}$ as in (12) sends the basis of characters in $\mathbb{F}_{p}(\widetilde{\Lambda}, \mathbb{K})$ to a basis of elementary functions (15), which is diagonalizing for the endomorphism $\operatorname{Fragm}_{n}(\tau) \in \operatorname{End}_{\Lambda}\left(\mathcal{F}_{p}\left(\Lambda, \mathbb{A}^{n}\right)\right)$.

Example 5.6. In the same setting as in 5.5, let us consider an endomorphism

$$
\Delta_{n, \varphi}=\varphi\left(\operatorname{Fragm}_{n}(\tau)\right)=\operatorname{Fragm}_{n}(\varphi(\tau)) \in \operatorname{End}_{\Lambda}\left(\mathcal{F}\left(\Lambda, \mathbb{A}^{n}\right)\right)
$$

commuting with shifts, where $\varphi \in \mathbb{K}\left[t, t^{-1}\right]$ is a Laurent polynomial. The matrix of Laurent polynomials that corresponds to $\Delta_{n, \varphi}$ is

$$
\widehat{A}_{\Delta_{n, \varphi}}=\varphi(\widehat{A})=\varphi\left(\widehat{A_{\operatorname{Fragm}_{n}}(\tau)}\right)=\widehat{A_{\operatorname{Fragm}_{n}(\varphi(\tau))}},
$$

where $\widehat{A}$ is as in 5.5. Recall that the equation $\Delta_{n, \varphi}(f)=0$ for $f \in \mathcal{F}_{p}\left(\Lambda, \mathbb{A}^{n}\right)$ is equivalent to

$$
\varphi\left(\widehat{A}\left(z^{-1}\right)\right)(\widehat{f}(z))=0 \quad \forall z \in \mathbb{K}^{\times} .
$$

By the Spectral Mapping Theorem, for every $z \in \mathbb{K}^{\times}$we have

$$
\operatorname{spec}\left(\varphi\left(\widehat{A}\left(z^{-1}\right)\right)\right)=\varphi\left(\operatorname{spec}\left(\widehat{A}\left(z^{-1}\right)\right)\right)=\left\{\varphi\left(\xi_{1}^{-1}\right), \ldots, \varphi\left(\xi_{n}^{-1}\right)\right\}
$$

where as before $\left\{\xi_{1}, \ldots, \xi_{n}\right\}=\{\sqrt[n]{z}\}$. The basis (15) is diagonalizing for $\Delta_{n, \varphi}$. Namely,

$$
\Delta_{n, \varphi}\left(v_{i} \cdot z^{\lambda}\right)=\varphi\left(\xi_{i}^{-1}\right) \cdot v_{i} \cdot z^{\lambda} .
$$

Indeed,

$$
\begin{gathered}
A_{\varphi\left(\operatorname{Fragm}_{n}(\tau)\right)}\left(z^{-1}\right) \cdot \widehat{v_{i} \cdot z^{\lambda}}=A_{\varphi\left(\operatorname{Fragm}_{n}(\tau)\right)}\left(z^{-1}\right) \cdot v_{i} \cdot \delta_{z}=\varphi\left(\widehat{A}\left(z^{-1}\right)\right)\left(v_{i}\right) \cdot \delta_{z} \\
=\varphi\left(\xi_{i}^{-1}\right) \cdot v_{i} \cdot \delta_{z}=\varphi\left(\xi_{i}^{-1}\right) \cdot \widehat{v_{i} \cdot z^{\lambda}}
\end{gathered}
$$

The Floquet-Fermi levels of $\Delta_{n, \varphi}$ in $\mathbb{T}^{1}=\mathbb{K}^{\times}$are

$$
\Sigma_{\Delta_{n, \varphi}, \mu}=\left\{\xi^{n} \in \mathbb{K}^{\times} \mid \varphi\left(\xi^{-1}\right)=\mu\right\}=\iota\left(\varphi^{-1}(\mu)\right)^{n}=\left(\varphi^{-1}(\mu)\right)^{-n} .
$$

In particular, the symbolic variety of $\Delta_{n, \varphi}$ is

$$
\Sigma_{\Delta_{n, \varphi}}=\left\{\xi^{n} \in \mathbb{K}^{\times} \mid \varphi\left(\xi^{-1}\right)=0\right\} .
$$


Letting $\varphi=q / z^{\beta}$, where $q=\prod_{j=1}^{d}\left(t-\alpha_{j}\right)^{k_{j}} \in \mathbb{K}[t]$ with $q(0) \neq 0$, we obtain

$$
\begin{gathered}
0 \in \operatorname{spec}\left(\varphi(\widehat{A})\left(z^{-1}\right)\right) \Longleftrightarrow \exists i: \varphi\left(\xi_{i}^{-1}\right)=0 \Longleftrightarrow \exists i: q\left(\xi_{i}^{-1}\right)=0 \\
\Longleftrightarrow \exists i: \xi_{i}=\alpha_{j}^{-1} \text { for some } j \in\{1, \ldots, d\} \Longleftrightarrow z \in\left\{\alpha_{1}^{-n}, \ldots, \alpha_{d}^{-n}\right\},
\end{gathered}
$$

where as before $\xi_{i}^{n}=z \in \mathbb{K}^{\times}, i=1, \ldots, n$. Note that the sequence $\alpha_{j}, \alpha_{j}^{2}, \ldots$ is periodic with period $d_{j}=\operatorname{ord}_{\mathbb{K} \times}\left(\alpha_{j}\right)$ coprime to $p$.

The kernel $\operatorname{ker}\left(\Delta_{n, \varphi}\right)$ is spanned by elementary vector functions $v_{i} \cdot z^{\lambda}$ as in (15) with $z \in$ $\left\{\alpha_{1}^{-n}, \ldots, \alpha_{d}^{-n}\right\}$. Choose $l \in\left\{0, \ldots, d_{j}-1\right\}$ such that $l \equiv n \bmod d_{i}$. Letting $z_{j l}=\alpha_{j}^{-l}=\xi_{i}^{l}$, such an elementary vector function is proportional to the vector function

$$
f_{j l}=z_{j l}^{\lambda} \cdot v_{i}=\left(\ldots, z_{j l}^{-1} v_{i}, v_{i}, z_{j l} v_{i}, z_{j l}^{2} v_{i}, \ldots\right) \in \mathcal{F}_{p}\left(\Lambda, \mathbb{A}^{n}\right),
$$

where $v_{i} \in \mathbb{A}^{n}$ is an eigenvector of the matrix $\widehat{A}\left(z_{j l}^{-1}\right)$ as in (13) with eigenvalue $\alpha_{j}=\xi_{i}^{-1}$. Actually $f_{j l}=\operatorname{fragm}_{n}\left(\alpha_{j}^{-\lambda}\right)$, where the character $\alpha_{j}^{-\lambda}=\xi_{i}^{\lambda}: \tilde{\Lambda} \rightarrow \mathbb{K}^{\times}$of order $d_{j}$ (equal to its period) does not depend on $n$.

Let us illustrate the latter issue on a concrete example.

Example 5.7. Suppose that $p \neq 3$. Consider the equation

$$
\Delta_{n, \varphi}(f)=\varphi\left(\operatorname{Fragm}_{n}(\tau)\right)(f)=\operatorname{Fragm}_{n}(\varphi(\tau))(f)=0,
$$

where $\varphi(t)=t+1+t^{-1}$. The roots $\alpha_{1}=\omega$ and $\alpha_{2}=\omega^{-1}$ of $\varphi$, where $\omega \in \mathbb{K}^{\times}$is a primitive cubic root of unity, have multiplicative orders $d_{1}=d_{2}=3$. There exists an elementary solution $z^{\lambda} \cdot v$ of (16) if and only if $z \in\left\{\omega^{n}, \omega^{-n}\right\}$, respectively $\xi \in\left\{\omega^{-1}, \omega\right\}$. Assuming that $n \equiv 0 \bmod 3$ we obtain $l=0$ and $z=1$. Letting $\xi=\omega^{-1}$ yields an elementary solution

$$
f=(\ldots, v, v, v, \ldots), \quad \text { where } \quad v=\left(\begin{array}{c}
1 \\
\omega^{-1} \\
\omega \\
1 \\
\vdots \\
\omega^{-1} \\
\omega
\end{array}\right) \in \mathbb{A}^{n} .
$$

This amounts to a 3-periodic solution

$$
\widetilde{f}=\left(\quad \ldots, 1, \omega^{-1}, \omega, 1, \omega^{-1}, \omega, \ldots\right) \in \mathcal{F}_{p}(\tilde{\Lambda}, \mathbb{K})
$$

of the scalar equation

$$
\Delta_{a}(f)=\varphi(\tau)(f)=0 \quad \text { with } \quad a=\delta_{-1}+\delta_{0}+\delta_{1} \in \mathbb{K}[\tilde{\Lambda}],
$$

where as before $\tilde{\Lambda}=\mathbb{Z}$. The latter solution does not depend on $n$. Any other elementary solution can be obtained from this one by a shift and inversion $\iota: \omega \longmapsto \omega^{-1}$. Actually every elementary solution of (17) is proportional either to $\widetilde{f}(\lambda)$ or to $\widetilde{f}\left(\lambda^{-1}\right)$. Thus the subspace of all $\Delta_{a}$-harmonic functions

$$
\operatorname{ker}\left(\Delta_{a}\right)=\operatorname{span}(\tilde{f}, \tilde{f} \circ \iota) \subseteq \mathcal{F}_{p}(\tilde{\Lambda}, \mathbb{K})
$$

is two-dimensional. It is easy to check that the same holds for every $p \neq 3$ and $n \not \equiv 0 \bmod p$.

Example 5.8. For any $p \neq 2$ we consider the lattice $\widetilde{\Lambda}=\mathbb{Z}^{2}$, its sublattice $\Lambda=2 \mathbb{Z} \times 2 \mathbb{Z} \subseteq \widetilde{\Lambda}$ of index 4 , and the commuting basic shifts $\tau_{1}=\tau_{(-1,0)}$ and $\tau_{2}=\tau_{(0,-1)}$ on $\widetilde{\Lambda}$. In the standard basis 
$\left(e_{00}, e_{10}, e_{01}, e_{11}\right)$ in $\mathbb{A}^{4}$ the corresponding matrices of Laurent polynomials $\widehat{A_{i}}=\widehat{A_{\operatorname{Fragm}(}\left(\tau_{i}\right)}, i=1,2$, are as follows:

$$
\widehat{A_{1}}\left(z^{-1}\right)=\left(\begin{array}{cccc}
0 & z_{1}^{-1} & 0 & 0 \\
1 & 0 & 0 & 0 \\
0 & 0 & 0 & z_{1}^{-1} \\
0 & 0 & 1 & 0
\end{array}\right) \quad \text { resp. } \widehat{A_{2}}\left(z^{-1}\right)=\left(\begin{array}{cccc}
0 & 0 & z_{2}^{-1} & 0 \\
0 & 0 & 0 & z_{2}^{-1} \\
1 & 0 & 0 & 0 \\
0 & 1 & 0 & 0
\end{array}\right) .
$$

In the common basis of eigenvectors

$$
v_{1}=\left(\begin{array}{c}
1 \\
x \\
y \\
x y
\end{array}\right), \quad v_{2}=\left(\begin{array}{c}
-1 \\
x \\
-y \\
x y
\end{array}\right), \quad v_{3}=\left(\begin{array}{c}
-1 \\
-x \\
y \\
x y
\end{array}\right), \quad v_{4}=\left(\begin{array}{c}
1 \\
-x \\
-y \\
x y
\end{array}\right)
$$

these commuting matrices are reduced to the diagonal form

$$
\operatorname{diag}\left(x^{-1},-x^{-1}, x^{-1},-x^{-1}\right) \quad \text { resp. } \quad \operatorname{diag}\left(y^{-1}, y^{-1},-y^{-1},-y^{-1}\right),
$$

where $x^{2}=z_{1}$ and $y^{2}=z_{2}$.

Consider further an endomorphism $\rho$ commuting with shifts, where

$$
\rho=\varphi\left(\operatorname{Fragm}\left(\tau_{1}\right), \operatorname{Fragm}\left(\tau_{2}\right)\right)=\operatorname{Fragm}\left(\varphi\left(\tau_{1}, \tau_{2}\right)\right) \in \operatorname{End}_{\Lambda}\left(\mathcal{F}\left(\Lambda, \mathbb{A}^{4}\right)\right)
$$

and $\varphi \in \mathbb{K}\left[z_{1}, z_{2}, z_{1}^{-1}, z_{2}^{-1}\right]$ is a Laurent polynomial. The same argument as in 5.6 above shows that the basis of elementary vector functions

$$
\left(\lambda \longmapsto v_{j}(z) \cdot z^{\lambda} \mid z \in \mathbb{T}^{2}, j=1,2,3,4\right)
$$

is diagonalizing for $\rho$. More precisely, letting

$$
\varphi_{1}(x, y)=\varphi(x, y), \quad \varphi_{2}(x, y)=\varphi(-x, y), \quad \varphi_{3}(x, y)=\varphi(x,-y), \quad \varphi_{4}(x, y)=\varphi(-x,-y),
$$

by the Spectral Mapping Theorem we obtain

$$
\rho\left(v_{j} \cdot z^{\lambda}\right)=\varphi_{j}\left(x^{-1}, y^{-1}\right) \cdot v_{j} \cdot z^{\lambda}
$$

(cf. Theorem 4.7(c)). The Floquet-Fermi level- $\mu$ set of $\rho$ is

$$
\Sigma_{\rho, \mu}=\left\{z=\left(x^{2}, y^{2}\right) \in \mathbb{T}^{2} \mid \exists j \in\{1,2,3,4\}: \varphi_{j}\left(x^{-1}, y^{-1}\right)=\mu\right\}
$$

(see 4.6). The eigenspace of $\rho$ with eigenvalue $\mu$ is

$$
E_{\rho, \mu}=\operatorname{span}\left(v_{j} \cdot z^{\lambda} \mid z=\left(x^{2}, y^{2}\right) \in \mathbb{T}^{2}: \varphi_{j}\left(x^{-1}, y^{-1}\right)=\mu, j=1,2,3,4\right) .
$$

Moreover,

$$
\mathcal{F}_{p}\left(\Lambda, \mathbb{A}^{4}\right)=\bigoplus_{\mu \in \mathbb{K}} E_{\rho, \mu}
$$

In particular, the symbolic hypersurface of $\rho$ is

$$
\Sigma_{\rho}=\left\{z=\left(x^{2}, y^{2}\right) \in \mathbb{T}^{2} \mid \exists j \in\{1,2,3,4\}: \varphi_{j}\left(x^{-1}, y^{-1}\right)=0\right\} .
$$

The space $E_{\rho, 0}$ of all $\rho$-harmonic vector functions is

$$
\operatorname{ker}(\rho)=\operatorname{span}\left(v_{j} \cdot z^{\lambda} \mid z=\left(x^{2}, y^{2}\right) \in \mathbb{T}^{2}: \varphi_{j}\left(x^{-1}, y^{-1}\right)=0, j=1,2,3,4\right) .
$$




\section{References}

[Bou] N. Bourbaki: Éléments de mathématique. XIV. Première partie: Les structures fondamentales de l'analyse. Livre II: Algèbre. Chapitre VI: Groupes et corps ordonnés. Chapitre VII: Modules sur les anneaux principaux. Actualités Sci. Ind., no. 1179. Hermann et Cie, Paris, 1952.

[Co] L. Collatz: Spektren periodischer Graphen, Results Math. 1 (1979) 42-53.

$\left[\mathrm{Ku}_{1}\right]$ P. A. Kuchment: On the Floquet theory of periodic difference equations. Geometrical and algebraical aspects in several complex variables (Cetraro, 1989), 201-209, Sem. Conf., 8, EditEl, Rende, 1991.

[Ku $\left.\mathrm{Ku}_{2}\right]$ P. Kuchment: Quantum graphs. II. Some spectral properties of quantum and combinatorial graphs. J. Phys. A 38 (2005) 4887-4900.

[Ku 3 ] P. Kuchment: Floquet Theory for Partial Differential Equations. Basel: Birkhäuser, 1993.

[KuPi] P. Kuchment, Y. Pinchover: Liouville theorems and spectral edge behavior on abelian coverings of compact manifolds. Trans. Amer. Math. Soc. 359 (2007) 5777-5815.

[KuVa] P. Kuchment, B. Vainberg: On the structure of eigenfunctions corresponding to embedded eigenvalues of locally perturbed periodic graph operators. Comm. Math. Phys. 268 (2006) 673-686.

[LN] R. Lidl, H. Niederreiter: Introduction to finite fields and their applications. Cambridge University Press, Cambridge, 1994.

[Ni] P. J. Nicholson: Algebraic theory of finite Fourier transforms, J. Comput. System Sci. 5 (1971) 524-547.

[vB] J. von Below: The index of a periodic graph. Results Math. 25 (1994), 198-223.

[Za $]$ M. Zaidenberg: Periodic binary harmonic functions on lattices. Adv. in Appl. Math. 40 (2008) 225-265.

$\left[\mathrm{Za}_{2}\right]$ M. Zaidenberg: Convolution equations on lattices: periodic solutions with values in a prime characteristic field, Geometry and Dynamics of Groups and Spaces. In Memory of Alexander Reznikov. Progr. Math., 265, 719-740, 2007, Birkhäuser, Basel.

[Za3] M. Zaidenberg: Periodic harmonic functions on lattices and points count in positive characteristic. Cent. Eur. J. Math. 7(3) (2009) 365-381.

Universit Grenoble I, Institut Fourier, UMR 5582 CNRS-UJF, BP 74, 38402 St. Martin d’Hères CÉdex, France

E-mail address: zaidenbe@ujf-grenoble.fr 